\title{
Luís Bueno
}

\section{Segundas Estórias: Eine andere Lektüre von «Famigerado»}

\begin{abstract}
Doch ist es möglich, daß es mir nicht nach Wunsch gelang, ihn zu täuschen, und daß er mit mir dasselbe Spiel trieb wie ich mit ihm. Wenn dies der Fall war, dann hat er, finde ich, seine Rolle gut gespielt, denn ich hatte den Eindruck, daß er mir nicht mehr mißtraute.

Oder aber, wer seine Rolle gut spielte, war ich, falls ich ihn überzeugt habe, daß ich gekommen sei, um zu politisieren. Wenn er das dachte, war er verrückt. Wahrscheinlich dachte er es nicht. Vielleicht dachte er es, weil er sich täuschen ließ und mich für aufrichtig hielt. So ging es wenigstens bei mir.

(Graciliano Ramos, S. Bernardo) $^{1}$
\end{abstract}

Ausgehend von der Hypothese, dass die Geschichten der Primeiras Estórias (dt. Erste Erzählungen) $)^{2}$ die nie veröffentlichten Segundas Estórias (dt. Zweite Erzählungen) beinhalten, schlägt dieser Artikel eine Lektüremethode für das Buch als Ganzes vor, die anhand der Analyse von «Famigerado» (dt. «Berüchtigt») $)^{3}$ veranschaulicht wird.

\section{Ein Rätsel, kein Mysterium}

In seinem berühmten Text über Tutaméia erzählt Paulo Rónai, er habe Guimarães Rosa eine Frage gestellt, die alle Leser gerne gestellt hätten:

- Warum Terceiras Estórias (dt. Dritte Erzählungen) ${ }^{4}$ - fragte ich ihn - wenn es keine zweiten gab?

- Einige sagen: Weil sie nach einer Gruppe anderer Geschichten geschrieben wurden, die nicht in die Primeiras Estórias (dt. Ersten Erzählungen) integriert wurden. Andere sagen: Weil der abergläubische Autor sich selbst verpflichten und die Möglichkeit schaffen woll-

1 Übersetzung aus: Graciliano Ramos: São Bernardo. Aus dem Portugiesischen von Wilhelm Keller. München: Carl Hanser 1965, S. 22.

2 Der deutsche Titel in der Übersetzung von Curt Meyer-Clason lautet Das dritte Ufer des Flusses: Erzählungen. Im Folgenden wird dieses Werk zur Übersetzung der zitierten Abschnitte aus Rosas Primeiras Estórias herangezogen und wie folgt gekennzeichnet: Übersetzung Meyer-Clason. Vollständige Literaturangabe: João Guimarães Rosa: Das dritte Ufer des Flusses. Erzählungen. Übersetzt von Curt Meyer-Clason. München: dtv 1975.

3 Portugiesischer Originaltitel: «Famigerado». Übersetzung Meyer-Clason.

4 Portugiesischer Originaltitel: Terceiras Estórias. Übersetzung Meyer-Clason.

Ә Open Access. (C) 2020 Luís Bueno, publiziert von De Gruyter. (c) BY-NC-ND Dieses Werk ist lizenziert unter der Creative Commons Attribution-NonCommercial-NoDerivatives 4.0 Lizenz.

https://doi.org/10.1515/9783110677713-007 
te, einen weiteren Band mit Erzählungen herauszugeben, die dann die Segundas Estórias (dt. Zweite Erzählungen) wären.

- Und was sagt der Autor?

- Der Autor sagt nichts - antwortete Guimarães Rosa mit dem Lachen eines großen Jungen, froh, den Kollegen in eine Falle gelockt zu haben. ${ }^{5}$

Es ist nicht sehr befriedigend, alle Fragenden in eine Falle zu locken. Aber das Fehlen einer präzisen Antwort lässt die Frage selbst nicht närrisch oder unfruchtbar erscheinen. Tatsächlich stehen wir vor einem weiteren Rätsel im Werk von Guimarães Rosa. Wie jede Frage, wenn auch oft vergebens, eine Antwort ersehnt, erfordert jedes Rätsel eine Lösung. Von diesem Rätsel hingerissen zu sein, kann auch ein Zeichen für etwas anderes sein, hinsichtlich dessen José Antonio Pasta Júnior in einer Abhandlung über Grande sertão: veredas folgendes beobachtet hat:

Es ist, als ob dieses Buch eigens durch seine Beschaffenheit und durch den mit seinem Leser geschlossenen Pakt die ästhetisch-literarische Gattung des Rätsels überschreitet, was dennoch auch die seine bleibt, um jene magisch religiöse, mysteriöse zu sein. Wie man weiß, verlangen Rätsel nach einer Lösung. Mysterien jedoch lassen nur Kult und Zelebrierung zu. ${ }^{6}$

Nicht nur Grande sertão: veredas, das gesamte Werk von Guimarães Rosa war und ist Kultobjekt. Seine Rätsel tendieren dazu, sich in Mysterien und Sakrilege zu verwandeln oder auch als ausgemachte Dummheiten aufgefasst zu werden. Diese Rätselhaftigkeit, deren Enthüllung unzugänglich erscheint, verweist auf einen absoluten Wahrheitsgehalt, der jede Offenbarung charakterisiert.

In diesem Aufsatz möchte ich eine mögliche Lösung vorschlagen und damit eine Methode des Lesens des gesamten Bandes der Primeiras Estórias ausarbeiten, die davon ausgeht, dass sich die Lösung des Rätsels im Buch selbst befindet. Da es nicht möglich ist, die gesamten Primeiras Estórias im Rahmen eines Artikels zu behandeln, wird die Geschichte «Famigerado» analysiert, sodass einerseits der Lösungsvorschlag besser zur Geltung kommt und andererseits die Gültigkeit der Lektüremethode, die daraus hervorgeht, demonstriert werden kann.

5 Paulo Rónai: Especulações sobre Tutaméia. In: Pois é: ensaios. Rio de Janeiro: Nova Fronteira 1990, S. 13-21, hier S. 14.

6 José Antonio Pasta Júnior: O romance de Rosa: temas do Grande sertão e do Brasil. In: Novos Estudos Cebrap 5 (1999), S. 61-70, hier S. 61-62 (unsere Übersetzung, Anm. d. Üb.). 


\section{Lösung}

Um direkt auf den Punkt zu kommen: Die Segundas Estórias wurden nicht veröffentlicht, weil sie bereits in den Primeiras Estórias enthalten sind. Das heißt, die Segundas Estórias wurden bereits veröffentlicht. Dies bedeutet wiederum, dass die Geschichten, aus denen der Band besteht, so strukturiert sind, dass sie zwei Geschichten beinhalten. Man könnte einwenden, dass viele Erzählungen auf diese Weise gelesen werden können. Zum Beispiel wenn man diejenigen in der ersten Person betrachtet, in denen ein wenig vertrauenswürdiger Erzähler seine Geschichte auf eine bestimmte Weise erzählt, um dadurch eine andere Geschichte zu verbergen, die aber auch erzählt wird - solange der Leser aufmerksam genug ist.

Die Besonderheit der Primeiras Estórias ist, dass das Werk vollständig auf diese Weise gelesen werden kann, das heißt, dass es sich um ein Element der Komposition handelt, das im Mittelpunkt der dort versammelten Geschichten steht. Am spezifischsten für die Verwendung dieser Technik im Buch ist die Verwicklung von Perspektiven, das konstante Spiel von Erscheinungen, und zwar nicht nur von ihnen und von dem, was sie enthüllen wollen, sondern auch von dem, was sie durch ihre Enthüllung letztendlich offenbaren.

Um zu verstehen, was hier gemeint ist, ist es interessant, zwei Geschichten zu betrachten, in denen diese Duplizität besonders deutlich hervortritt, da sie sich regelrecht auf der Oberfläche des Textes befindet.

Die erste davon ist «Nada e nossa condição» (dt. "Nichts und unser Zustand») ${ }^{7}$, eine Geschichte, in der die Kritiken in gewisser Hinsicht bereits auf das hingewiesen haben, was wir hier als Duplizität der Geschichten bezeichnen. ${ }^{8}$ Das Projekt des Erzählers besteht darin, einen Onkel zu verherrlichen:

In meiner Familie, in meiner näheren Heimat, hat keiner jemals einen Mann gekannt, der, eher von Auszeichnungen als von Aussehen, der alte König oder jüngere Prinz in den künftigen Märchen hätte sein können. Er war Gutsbesitzer und nannte sich Onkel Brud'Antônio. ${ }^{9}$

Jedoch scheitert dieses Vorhaben kläglich zur gleichen Zeit als es sich konkretisiert, da dieser Erzähler, blind von der Bewunderung für den alten Verwandten

7 Übersetzung Meyer-Clason.

8 Siehe in diesem Zusammenhang das Kapitel «Tagträume eines Patriarchen» in Ana Paula Pacheco: O lugar do mito. Narrativa e processo social das Primeiras estórias de Guimarães Rosa. São Paulo: Nankin 2006 und Volnei EdsonSantos/AdelaideCaramuru Cezar: Patriarcalismo em «Nadae a nossa condição» de João Guimarães Rosa. In: Volnei Edson Santos (Hg.): Sopros do silêncio. Londrina: Eduel 2008, S. 11-31. Hier gibt es verschiedene Ansätze, die auf die gleiche Duplizität hinweisen. 9 João Guimarães Rosa: Primeiras Estórias. Rio de Janeiro: José Olympio ${ }^{6} 1972$, S. 80. Übersetzung Meyer-Clason, S. 71. 
und durch seine Prinzipientreue gegenüber den alten Werten, die ihm diese Bewunderung erlauben, letztendlich eine andere Geschichte erzählt als die, die er eigentlich erzählen will. Was er bewusst zeichnet, ist die Figur eines großzügigen und umsichtigen Mannes, der nach dem Tod seiner Frau seinen Reichtum erweitert, sich um die Zukunft seiner Töchter kümmert: «Er selber gab mit seinem verdienten Geld vor, seine Ländereien gültig zu verkaufen; mit Geld, das er pünktlich an die Töchter und Schwiegersöhne sandte.» ${ }^{10}$ Direkt im Anschluss sollte die großzügigste und unverstandenste Geste gemacht werden, um zu geben, was ihm am kostbarsten war: «unter seine zahlreichen barfüßigen Diener, schwarze, weiße, braune, Mulatten, Niemande, Jemande, Landarbeiter, Viehtreiber und Taglöhner, die nächsten, die nie aufbegehrt hatten [...] verschenkte und verteilte Onkel Brud'Antônio seine Ländereien.»" ${ }^{11}$

Aber das Vertrauen dieses Erzählers in die Überlegenheit nicht nur seines Onkels, sondern seiner ganzen Familie im Vergleich zu den Bediensteten, diese Niemande und Jemande, lässt eine andere Geschichte sichtbar werden. Er zeigt die Abwesenheit der Dankbarkeit von denen auf, die das Land erhalten haben und protestiert dagegen:

Sie taten so, als seien sie die Herren, sie, die anderen gewöhnten sich daran. Sie begriffen ihn nicht. Sie liebten ihn sicherlich schon deshalb nicht, weil sie stets seine undurchsichtige Gestalt zu fürchten und seinen Einfluß zu achten hatten; in seinem burgartigen Bau war er nach wie vor ein Herrscher. ${ }^{12}$

Der Leser, der der Geschichte an dieser Stelle folgt, fragt: Wie, «also taten sie so, als wären sie Meister»? Wurde ihnen denn das Land nicht gegeben? War es nicht zu Recht ihres? Und außerdem: Woher kommt diese Erhabenheit, von der Größe des Menschen oder von der Tatsache, dass er fast das ganze Land gegeben hat, sich aber das Herrenhaus vorbehält, das hoch oben liegt, von wo aus er über alle wacht?

Diese Fragen können gestellt werden, weil der Erzähler durch die Annahme einer rigiden richtig-falsch-Dualität die Perspektive der «undankbaren Nutznießer> der Großzügigkeit des alten Verwandten, die in jeder Hinsicht von seiner eigenen abweicht, eröffnet, und zwar indem er diese verurteilt.

Neben der Figur der ersten Geschichte, der des großzügigen Mannes, der das teilt, was er hat, erhebt sich die dominierende Figur der zweiten Geschichte, eine Figur, die jetzt überschüssige Güter teilt, da sich ihr Vermögen vermehrt und sich

10 Ebda., S. 86. Übersetzung Meyer-Clason, S. 77.

11 Ebda., S. 86. Übersetzung Meyer-Clason, S. 77.

12 Ebda., S. 87. Übersetzung Meyer-Clason, S. 78. 
in die Stadt verlagert hat, in der die Töchter leben, ohne die symbolische Position des alten Besitzers und Herrn aufzugeben, der alle Begünstigten einer ständigen Kontrolle unterwirft. Ana Paula Pacheco versucht, diese Dualität zusammenzufassen, wenn sie sagt, es handele sich um «einen Erzähler, dem andere Stimmen widersprechen, die im Diskurs gespannt bestehen bleiben, wenn sie auch am Ende verstummen». ${ }^{13}$

Die Situation ist weniger ein Widerspruch als die Koexistenz von zwei Logiken in einer besonders komplexen historischen Erfahrung wie der Modernisierung, besonders im problematischen brasilianischen Fall. Die eine, die in der ersten Erzählung bevorzugt wird, ist die herrschaftliche, die Onkel Brud'Antônio zu erhalten sucht, indem er verbarrikadiert bleibt, um über alles zu wachen, und die in den Augen seines Neffen, des Erzählers, wirksam bewahrt wird. Die andere, die in der zweiten Geschichte auftaucht, jene von denen, die ihn «sicherlich haßten [...], tausendjährig und tierisch» ${ }^{14}$ und diese Erhabenheit zurückweisen, ist die moderne Logik, die Gleichheit zwischen Individuen herstellt.

Die zweite Geschichte, über die etwas ausführlicher gesprochen werden muss, ist «A benfazeja» (dt. «Die Wohltäterin»). ${ }^{15}$ In ihr hängt die Gestaltung des Erzählers, der sich wegen der Art und Weise, wie alle dort über eine Frau, die Mula-Marmela, urteilen, an eine ganze Gemeinschaft wendet, vom Zusammenleben zweier Geschichten ab. Die erste ist diejenige, die die Gemeinschaft geschaffen hat; die zweite, diejenige auf die der Erzähler den Anspruch erhebt, die wahre zu sein. Für die Gemeinde ist die Frau eine schlechte Frau, die ihren Ehemann getötet hat und, wie man vermutet, den Stiefsohn. Der Erzähler hingegen entlockt dieser Mordhandlung eine andere Bedeutung, in der die Morde selbst, nebst der Vergiftung, die den Stiefsohn Jahre vor seinem Tod erblinden lassen hatte, keine Gesten der Bosheit waren. Sie sind eher Zeichen der Entsagung - denn sie hatte sie geliebt - und der Sorge um das Gemeinwohl, da die Toten hoffnungslose Teufel waren.

Die Erzählweise der beiden Geschichten erzeugt eine große Dissonanz. Der Erzähler stellt eine Reihe von Fragen: «Und ihr, wollt ihr noch immer diese Frau beschuldigen, die Marmela, sie verurteilen, sie abstoßend finden? Laßt sie in Ruhe, wenn ihr sie nicht versteht und ihn [den Stiefsohn] noch weniger. Ein jeder mit seiner Niedertracht, ein jeder mit seiner Hochherzigkeit». ${ }^{16}$ Mit dieser Haltung schafft er eine unüberwindliche Distanz zwischen dem, der von außen kommt,

13 Pacheco, Ana Paula: O lugar do mito: Narrativa e processo social nas «Primeiras Estórias» de Guimarães Rosa. São Paulo: Nankin 2006, S. 217-218 (unsere Übersetzung, Anm. d. Üb.).

14 Rosa: Primeiras Estórias, S. 88. Übersetzung Meyer-Clason, S. 79.

15 Übersetzung Meyer-Clason.

16 Rosa: Primeiras Estórias, S. 131. Übersetzung Meyer-Clason, S. 115. 
dem Erzähler selbst, jemandem, der in der Lage ist, Mula-Marmela zu verstehen, und allen anderen, denen aus dem Ort, die sich in all den Jahren, in denen sie dort lebte nicht einmal die Mühe machten, ihren Namen zu kennen und sie ausschlossen.

Solch unterschiedliche Urteile deuten auf unterschiedliche Wertsysteme hin. Aber diese Unterscheidung ist nur Schein, denn die Werte, die der Erzähler hervorruft, um die Größe von Mula-Marmela aufzuzeigen sind nur teilweise weniger konventionell. Ja, sie ist eine arme Frau, der niemand Aufmerksamkeit schenkt. Er hingegen versucht, sie als Individuum zu sehen und spricht wirklich mit ihr, ist kurz davor, all ihre Handlungen offenlegen zu können, die niemand ihr zuschreiben konnte, wie die, den Stiefsohn blind zu machen. Auf der anderen Seite ist das, was er benutzt, um Marmelas Verhalten zu rechtfertigen und Großzügigkeit und Selbstaufopferung als Motor von allem zu bestimmen, etwas, das absolut der Logik derer entspricht, die er beschuldigt:

Und ihr altes Verbrechen? Ich habe immer gehört, der von ihr Ermordete war ein Wüterich, ein Hund von einem Mann, das Entsetzlichste an Verhängnis, Gefahr und Strafe für die Ortsbewohner. Aus dem, was ich von euch selbst gehört habe, entnehme ich, daß ihr dafür alle mächtig in ihrer Schuld steht, wenn euch das auch nicht zum Bewußtsein gekommen zu sein scheint und ihr diese Dankbarkeit auch nicht zeigt. ${ }^{17}$

Wenn man im Sinne der bildhaften Darstellung denkt, die das gesamte Buch der Primeiras Estórias mit der brasilianischen Modernisierung vornimmt, ist es nicht schwer, eine Sackgasse aufzuzeigen: Der Erzähler, der die Gemeinschaft des Konventionalismus beschuldigt, tut dies innerhalb der konventionellen Logik. Was die Intervention eines modernen Gedankens zu sein scheint, der in den Handlungen eines jeden Individuums und nicht in den Ursprüngen oder in der Klassenpositionierung den Wert jedes Einzelnen entdecken will, beruht auf einer alten Ordnung, in der blinde Gerechtigkeit praktiziert wird, der alten Logik, dass ein «guter Verbrecher ein toter Verbrecher ist», wobei es keine Rolle spielt, ob dies im Gesetz vorgesehen ist oder nicht - und das ist es nicht.

Wie gesagt, verursacht die Vorgehensweise der zwei Geschichten jedoch eine tiefe Vermischung von Perspektiven und ein Spiel von Erscheinungen

Eine Analogie mit Maupassants Boule de suif (dt. Dickchen)» ${ }^{18}$ kann helfen, diesen grundlegenden Punkt zu verdeutlichen. Dort zeigt ein klassischer Erzähler

17 Ebda., S. 126. Übersetzung Meyer-Clason, S. 110.

18 Im Folgenden zum Zitieren verwendete Übersetzung ins Deutsche: Guy de Mauspassant: Dickchen. Aus dem Französischen übersetzt von Georg Freiherrn von Ompteda. Berlin: Egon Fleischel \& Co 1910. Verfügbar unter http://gutenberg.spiegel.de/buch/dickchen-2530/2. Letzter Zugriff: 8.9.2018. Wie folgt gekennzeichnet: Übersetzung Ompteda, o. S. 
der dritten Person einen Akt der Großzügigkeit des Titelcharakters. Jedoch wird diese Handlung von den anderen Charakteren nicht als großzügig verstanden, außer natürlich, wenn sie von dieser profitieren und es notwendig wird, das Mädchen von deren Ausführung zu überzeugen.

Die Handlung ist wohlbekannt. Eine Gruppe von Menschen reist, eine ruhige Periode im Deutsch-Französischen Krieg von 1870 ausnutzend, in einem «große[n] Postwagen mit vier Pferden bespannt» ${ }^{19}$ durch Frankreich. Es handelt sich um eine sehr repräsentative Gruppe, da ihre Mitglieder aus dem Großbürgertum, dem Adel, zwei Nonnen und zwei weiteren Personen bestehen, einem Jungen, der als «Schrecken aller anständigen Menschen» ${ }^{20}$ bezeichnet wird, und der Titelfigur, die indirekt wie folgt beschrieben wird: «Sobald man sie erkannt hatte, tuschelten die ehrbaren Damen miteinander und die Worte 〈Prostituierte〉 und 〈Schandfleck〉 fielen so laut, daß Dickchen den Kopf hob.» ${ }^{21}$ Die Gruppe wird in einem Wirtshaus der dritten Klasse aufgehalten und an der Weiterreise gehindert, weil der befehlsgebende preußische Kommandant die junge Frau begehrt, die die anderen als verkommen betrachten. Es stellt sich heraus, dass sie eine Nationalistin ist, die bereits Ärger mit preußischen Soldaten gehabt hatte und sich weigert, der Aufforderung des Kommandanten nachzukommen. Verärgert darüber, dass sie im tiefsten Winter am Ende der Welt festsitzen, beginnen Dickchens Reisegefährten, das Mädchen dazu zu überreden, sich hinzugeben und so den Durchgang frei zu machen, woran sich auch die Gräfin beteiligt (die fragend den Weg bereitet) sowie die Nonnen (die antwortend vervollständigen):

«Sie meinen also, meine Schwester, daß Gott alle Mittel gutheißt und die That vergiebt, wenn nur der Beweggrund rein ist?»

«Wer wollte daran zweifeln, Frau Gräfin? Eine an und für sich böse That wird oft verdienstvoll durch den Gedanken, der in ihr lebt.» ${ }^{22}$

Kurzum: Der Druck ist enorm und das arme Mädchen gibt nach und bringt ein ihm fast unerträgliches persönliches Opfer, das die ganze Gruppe befreien könnte. Am nächsten Tag kommt ihr keinerlei Anerkennung bezüglich ihres Opfers zu. Im Gegenteil, die im Vorfeld geäußerte Idee, dass es bei ihr keinen Unterschied machen würde, da Dickchen sich ohnehin jedem Mann hingebe, drängt sich auf und die Behandlung, die ihr wiederfährt, ist noch kälter als zuvor, als ob das, was sie getan hat, um sie zu retten nur beweisen würde, wie verkommen sie war. Und auf

19 Mauspassant: Boule de Suif, Übersetzung Ompteda, o. S.

20 Ebda.

21 Ebda.

22 Ebda. 
diesem letzten Teil der Reise geben sie ihr nicht einmal ein wenig Essen ab, gerade ihr, die sie zuvor auf dem ersten Teil der Reise allesamt ernährt hat.

In dieser Erzählung wird das Spiel der Erscheinungen direkt durch eine Inversion der Werte wahrgenommen. Das edelste aller Geschöpfe - ein Edelmut, der durch Selbstaufopferung offenbart wird - wird von den anderen Geschöpfen für verabscheuenswert erachtet, die selbst verabscheuenswürdig sind, eben genau deshalb, da sie ihren persönlichen Nutzen sowohl über ihre kollektiven Werte (die Nation) als auch über die Überzeugungen einer anderen Person stellen. Zwei gegensätzliche Wertsysteme stehen sich gegenüber, die einer traditionellen bürgerlichen Moral voller vorgefasster Meinungen, und eines anderen Systems, definitiv moderner, das den Wert der Individuen anhand der Handlungen, die sie praktizieren, erkennt, ungeachtet ihrer sozialen Herkunft. Kann man sagen, dass wir in Dickchen auch zwei Geschichten finden müssen?

Nein, denn die beiden Wertesysteme scheinen sich gegenseitig aufzuheben, indem sie sich ergänzen. Wenn man die Lektüre des Textes verlässt, zweifelt niemand daran, dass die einzige Geschichte, die dort erzählt wird, die der Größe des Protagonisten und der Heuchelei der anderen ist. Der Unterschied der Perspektiven zwischen dem, was auf der einen Seite und was auf der anderen als abscheulich betrachtet wird, wird aufgehoben und zu einer Vision, in der klar erkennbar ist, wer abscheulich ist und wer nicht.

In «Die Wohtäterin» ergeben sich die Geschehnisse auf eine ganz andere Weise. Durch die zwei mit der anklagenden Stimme des Erzählers an die Oberfläche gebrachten Geschichten werden die beiden unterschiedlichen Perspektiven bewahrt. Wenn der Erzähler andererseits mit den Werten, denen er sich entgegenstellt, Kompromisse eingeht, indem er die Gültigkeit seiner Perspektive mit der gewaltsamen Logik der Perspektive der Gemeinschaft, an die er sich richtet und die er anklagt, rechtfertigt, verwickelt sich alles zunehmend und wird dadurch komplizierter. Hinzu kommt die Schwere der Taten der Mula-Marmela, die weit über die Angriffe auf eine konventionelle bürgerliche Moral hinausgehen: Sie tötet und macht blind.

In Dickchen werden wir dazu gebracht, nach Dingen wie «inwiefern sind die Mitreisenden besser als Dickchen?», oder gar «inwiefern ist der preußische Kommandeur schlechter als Dickchens Reisegefährten?» zu fragen. Aber niemand wird auf die Frage «ist Dickchen schlechter als ihre Mitreisenden oder der preußische Kommandeur?» gebracht. Nein. Dickchen ist besser als sie. Der Charakter repräsentiert die unzweifelhafte ethische Referenz (und kaum sichtbar für denjenigen, der Prostitution und Würde als unvereinbar beurteilt), die sowohl die Erzählung als auch den Kommentar strukturiert, den sie über diese Gesellschaft machen möchte.

In «A benfazeja» hingegen werden wir aufgefordert, auch so etwas zu fragen wie «inwiefern ist Marmela gleich besser als die Mörder, die sie getötet hat?», oder 
auch «inwiefern ist der Erzähler besser als die Bewohner jenes Ortes?». Aber wir fragen bloß, ohne schlüssige Antworten zu erhalten. Alle Formen der Argumentation, die auf der Dualität von Recht und Unrecht beruhen, sind unhaltbar. Wenn der Erzähler schließlich den drastischen Standpunkt einnimmt, Größe in den Verbrechen von Marmela zu sehen, weil sie die Allgemeinheit von zwei Übeltätern befreit hat, können wir ihn in der Tat wieder an diejenigen Werte annähern, gegen die er ankämpft. Aber es ist auch wahr, dass es sich dabei lediglich um eine rethorische Strategie handeln kann, um sowohl auf das stereotype Urteil, dass sie über Marmela fällen, als auch auf die bösartige Perspektive aufmerksam zu machen, die zu diesem Urteil führt. Das heißt, es ist möglich, dass das Projekt des Erzählers letztendlich nicht ist, diese Frau zu resozialisieren, aber durchaus, die Möglichkeit ihrer Resozialisierung aufzuzeigen, dass es notwendig ist, jedes Individuum, wodurch es auch charakterisiert ist, in seinen eigenen Begriffen $\mathrm{zu}$ verstehen. Und das ist möglich, solange man erkennt, dass das, was diese Gemeinschaft benutzt, um Marmelas moralische Verurteilung zu rechtfertigen (ihr ihre Verbrechen nicht zu vergeben), nur der Anschein von Gerechtigkeit ist - und von moderner Gerechtigkeit, die die Auge-um-Auge-Logik ablehnt. Denn niemand unternimmt rechtliche Schritte gegen die Mörderin: Sie marginalisieren sie lediglich, während sie die Vorteile ihres Verbrechens hinnehmen.

So verweist die Dualität der Geschichten einer Frau auf der Oberfläche des Textes in «A benfazeja» auf die Dualität des Erzählers selbst hin, sowie, wenn wir so wollen, auf die Ambiguitäten des brasilianischen Modernisierungsprozesses.

\section{3 «Famigerado»}

In Kurzgeschichten wie «Nada e nossa condição» und "A benfazeja» bieten sich die zwei Geschichten durch die ganz eigentümliche Beschaffenheit der Erzählstimme dem Leser mehr oder weniger direkt an. Aber das ist nicht immer der Fall. In vielen Geschichten der Primeiras Estórias sind die zweiten latent in den ersten enthalten und erfordern von dem Leser eine intensivere Interpretation. In «Famigerado» ist diez genau der Fall.

In diesem Text haben wir einen gebildeten Erzähler, der uns den unerwarteten und einzigartigen Charakter dessen ankündigt, was er erzählen wird: «Es kam zu einer ungewissen Gelegenheit - das Ereignis. ${ }^{23}$ Und dieses einzigartige Ereignis ist der Kontakt zwischen zwei entfernten Punkten der Gesellschaft: dem Arzt der kleinen Stadt und dem alten, unerbittlichen Jagunço. Die erste Geschichte ist

23 Rosa: Primeiras Estórias, S. 9. Übersetzung Meyer-Clason, S. 12. 
die «Sache [...] zum laut Lachen» ${ }^{24}$, der scherzhafte Fall, wie der Arzt mit seinen Waffen des Gelehrten den Jagunço einwickelt. Die erste Geschichte ist die vom Sieg des Wortes über die Waffen, der Aufklärung über den Obskurantismus, des Modernen über das Archaische.

Es gibt sich, dass der Jagunço Damázio, Bewohner eines abgelegenen Ortes (ferne sechs Leguen oder vierzig Kilometer von der Stadt entfernt, in der der Arzt wohnt), von einem «jungen Mann von der Regierung» ${ }^{25}$, der sich kürzlich in diesen Banden niedergelassen hatte, berüchtigt genannt wird. Er weiß nicht, ob es sich um eine Beleidigung handelt oder nicht und beschließt, auf jenen zurückzugreifen, der es weiß, um seine Zweifel darüber auszuräumen. Der Arzt erschreckt sich natürlich über den Besuch des Mannes, erkennt dann aber doch, dass sich für ihn alles innerhalb der Grenzen des Angenehmen abspielen kann. Er macht sich die Mehrdeutigkeit des Wortes zunutze und entzieht sich der Situation, indem er versichert, dass berüchtigt keine Beleidigung sei und entgegnet: «So wie Sie mich hier vor sich sehen, möchte ich, hm, liebend gern bald mal eine Stunde lang berüchtigt sein - recht berüchtigt, und zwar so viel wie möglich...!» ${ }^{26}$ Damit täuscht er den Jagunço und rettet die Haut des jungen Mannes von der Regierung.

José Miguel Wisnik fasst in einer bekannten Analyse, die das Spiel zwischen modern und archaisch verdeutlichen möchte, das in den Primeiras Estórias zur Anwendung gebracht wird, diese Erzählung zusammen, indem er behauptet: «Man kann den Erzähler von «Famigerado» als einen Gelehrten Pedro Malasartes betrachten, oder seine Geschichte als etwas wie das unwahrscheinliche Duell des Jagunço mit einem 〈Doktor Jabuti.$^{27}{ }^{28}$ Ana Paula Pacheco distanziert sich nicht von dieser Lektüre, zieht daraus jedoch eine besondere Konsequenz, die auf eine Art Widerspruch in Guimarães Rosas Werk hinweist: «Das «zivilisierte Mundwerk〉 des Erzählers (das an die Fähigkeit des Schriftstellers erinnert, den Sertão für die

24 Ebda., S. 13. Übersetzung Meyer-Clason, S. 16.

25 Ebda., S. 11. Übersetzung Meyer-Clason, S. 14.

26 Ebda., S. 12. Übersetzung Meyer-Clason, S. 15.

27 Laut dem Autor des Artikels handelt es sich bei «Jabuti» um eine gemeine brasilianische Schildkrötenart, die in der brasilianischen Folklore als Volkscharakter bzw. einer Art Fabeltier in vielen Erzählungen vorkommt und dem als besonderes Merkmal Klugheit zugeschrieben wird, allerdings nicht im Sinne einer Gelehrten-Klugheit, sondern als einer angeborenen, listigen Schlauheit, ähnlich dem Fuchs in europäischen Fabeln. Die Jabuti-Schildkröte versteckt ihre ausgefuchste List hinter ihrer Erscheinung als einem langsamen und gleichgültigen Tier. Der vom Autor übernommene Ausdruck «Doktor Jabuti» (im pt. orig. Text jabuti doutoral) meint, dass der Doktor in der Erzählung von Guimarães Rosa vergleichbar mit der Figur der Jabuti-Schildkröte ist, im Unterschied zu den übrigen folklorischen Erzählungen hier allerdings als Gelehrter, als Doktor auftritt. (Anm. d. Üb.)

28 Wisnik, José Miguel. O famigerado. In: Scripta 5/10 (2000), S. 177-198, hier S. 186. Üb. i. Dt.: Unsere Übersetzung (Anm. d. Üb.). 
Stadt zu würdigen, durch den berühmten Stil sowie durch die Art und Weise der Darstellung der Welt des Sertão) scheint in dieser Geschichte gerade diesen anderen (Jagunço) herabzusetzen.» ${ }^{29}$

Diese Analysen betrachten nur die erste Erzählung von «Famigerado». Was, wenn wir in der zweiten Erzählung herausfinden, dass der überwältigende Sieg des Doktors über den Jagunço nicht die einzige Schlussfolgerung ist, die uns das Ereignis ermöglicht? Was, wenn wir uns fragen können, wer wohl der wahre Pedro Malasartes in diesem Fall ist?

Der Arzt erzählt von Ereignissen, die er beherrscht von der Angst erlebt, die Damázio in ihm hervorruft. Noch bevor er weiß, um wen es sich handelt, stellt er es sich vor: «[Er] konnte nur ein Schläger aus dem Sertão sein, ein Jagunço bis zum Schleim seiner Schnauze.» ${ }^{30}$ Und denkt: «Die Angst angelte mich.» ${ }^{31}$ Später, als sich der Mann vorstellt, bekommt er es noch einmal mit der Angst zu tun: «Zusammenzucken. Damázio, wer hatte nicht von ihm gehört? Der Bluthund aus Geschichten, léguasweit, mit Dutzenden von Toten auf dem Buckel, ein erzgefährlicher Gauner.» ${ }^{32}$ Nicht einmal die Beschwörung des allgemeinen Geredes, das besagt, er sei ruhiger geworden, kann ihn beruhigen: «Doch wer wollte dem Waffenstillstand eines Wüterichs vertrauen?» 33

Obwohl er die Erzählung lenkt, die im Nachhinein stattfindet, scheint alles darauf hinzuweisen, dass der Jagunço selbst derjenige ist, der die erzählten Handlungen lenkt. Wie es der Doktor letztendlich selbst anerkennt, ist «Angst [...] die äußerste Unwissenheit in stacheligen Momenten». ${ }^{34}$ Und der Erzähler scheint ein diffuses Bewusstsein für seine Unsicherheiten $\mathrm{zu}$ haben, schon dadurch, dass er Damázios Handlungen durchweg mittels Fragen charakterisiert («ob aus List?» ${ }^{35}$ ), durch Vermutungen («Lachte, und wie», «Und ob es ihm ernst war» $\left.{ }^{36}\right)^{37}$ oder der Verwendung des Konjunktivs («Waren wohl seine Gefangenen» ${ }^{38}$ ). ${ }^{39}$

29 Pacheco: O lugar do mito, S. 78. Üb. i. Dt.: Unsere Übersetzung.

30 Rosa: Primeiras Estórias, S. 9. Übersetzung Meyer-Clason, S. 12.

31 Ebda., S. 9. Übersetzung Meyer-Clason, S. 12.

32 Ebda., S. 10. Übersetzung Meyer-Clason, S. 13.

33 Ebda., S. 10. Übersetzung Meyer-Clason, S. 13.

34 Ebda., S. 9. Übersetzung Meyer-Clason, S. 13.

35 Ebda., S. 10. Übersetzung Meyer-Clason, S. 14.

36 Ebda., S. 10. Übersetzung Meyer-Clason, S. 14.

37 Aus der Originalübersetzung von Curt Meyer-Clason übernommen (s. ebda., S. 14). Im portugiesischen Originaltext heißt es «se é que se riu» bzw. «se sério, se era». Um der Textinterpretation von Luís Bueno an dieser Stelle gerecht zu werden, der von «Vermutungen» ausgeht, schlagen wir als alternative Übersetzung «wenn es ein Lachen war» und «wenn es ihm ernst war» vor. (Anm. d. Üb.)

38 Rosa: Primeiras Estórias, S. 9. Übersetzung Meyer-Clason, S. 12.

39 Im portugiesischen Originaltext wird der Annahmecharakter durch die Verwendung des Konjunktivs ausgedrückt: «seriam seus prisioneiros». (Anm. d. Üb.) 
Nicht nur das. Der Rhythmus der Ereignisse wird von Damázio bestimmt. Angesichts der ungewöhnlichen Situation kann der Arzt nur annehmen, was dieser Mann dort macht, und Damázio bestärkt ihn die ganze Zeit in seinen eigenen Annahmen, ohne irgendeine Erklärung. Zunächst zeigt er Respekt, indem er «ungeachtet des Brauchs» die Einladung ablehnt, einzutreten. ${ }^{40}$ Der Arzt stellt ihm daraufhin eine Frage und er erklärt zunächst, wozu er nicht gekommen ist: zur Konsultation. Er kündigt lediglich an: «Ich bin gekommen, um Sie um eine Meinung, um eine Erklärung zu bitten...» ${ }^{41}$ Aber die Frage, die ihn dorthin gebracht hat, wird weitestmöglich verzögert. Er steigt vom Pferd ab, was dem Doktor die Gelegenheit gibt, zu sehen, dass er bewaffnet ist, «und zwar mit gereinigten Waffen», ${ }^{42}$ dass der Sattel seines Pferdes teuer war, das Ergebnis einer vorzüglichen Arbeit, dass er aus dem Norden stammt, dass er stark ist «der ganze Mann ein Baumstamm.» ${ }^{43}$ Erst später stellt sich der Mann als Damázio von den Siqueiras vor. Was der Erzähler bei seiner ersten Annahme bereits vermutet hatte, bestätigt er ihm: es handelt sich um einen bekannten Tyrannen.

Nachdem er sich vorgestellt hat, kann er schließlich zur Frage kommen. Was er aber nicht tut. Zuerst beginnt er, von dem jungen Mann von der Regierung zu erzählen: «Sie müssen wissen, dass ich nichts mit ihm zu schaffen habe...» ${ }^{44}$ sagt er und unterbricht sich. Er wechselt das Thema: «Und sprach weichlich: von verschiedenen anderen Personen und Dingen, von der Serra, von São Ão, unzusammenhängendes, zähflüssiges Zeug». ${ }^{45}$ Aber er erzählt die Geschichte nicht weiter, er ändert das Thema, ohne sie zu Ende zu bringen. Kurzum, er windet sich sehr, weicht dem eigentlichen Thema aus, alles, um auf unerwartete Weise die Frage aufzuwerfen, so wie einer, der einen Schuss abfeuert, wie folgt beschrieben: «Und dann, bum: «Und nun tu mir Euer Gnaden das gute Werk an und erkläre mir, was folgendes eigentlich genau heißt: berichtigt - berechtigt - beträchtigt - bemächtigt?> Sagte sie in einem Schuß, die Worte, hatte sie zwischen den Zähnen hervorgestoßen.» ${ }^{46}$ Diese abrupte Einführung kann zu der Annahme führen, dass er es nun eilig hat, eine Erklärung zu erhalten. Aber nein. Er spricht weiter, eröffnet keine Möglichkeit für eine Antwort: Er erklärt, dass er von weither gekommen sei, dass es dort niemanden gebe, der jenes Problem lösen könne. Wörterbücher fehlten. Vielleicht könnten Priester eine Antwort geben, er verstehe sich jedoch nicht

40 Ebda, S. 10. Übersetzung Meyer-Clason, S. 12.

41 Ebda, S. 10. Übersetzung Meyer-Clason, S. 13.

42 Ebda.

43 Ebda.

44 Ebda, S. 11. Übersetzung Meyer-Clason, S. 14.

45 Ebda.

46 Ebda. 
mit Priestern, «die wickeln einen gleich ein». ${ }^{47}$ Der Arzt bemerkt diese Verzögerung: «Er wehrte meiner Antwort, wünschte nicht, daß ich sie sofort gab». ${ }^{48} \mathrm{Er}$ skizziert jedoch keine andere Reaktion, als sich selbst für klug genug zu erklären, um die Einstellung des Jagunço zu durchschauen.

Was er nicht bemerkt, ist, dass er von Damázio manipuliert wird. Der Jagunço gibt ihm Zeit, Vermutungen anzustellen. Und wie es in einer solchen Situation natürlich ist, führt die Angst angesichts der Möglichkeit des Ausbruchs der Gewalt und der Unkenntnis der Absichten des Gesprächspartners zu einer schrecklichen Annahme:

Und schon hob mich ein anderer schwindelerregender Schrecken ins Ungewisse: Einer konnte sich eine Schindluderei geleistet, konnte jenes für den Mann beleidigende Wort frei erfunden und mir in den Mund gelegt haben. Vielleicht war er deshalb hergekommen, um den starken Mann zu spielen und mir von Angesicht zu Angesicht die verhängnisvolle, die qualvolle Genugtuung abzuverlangen. ${ }^{49}$

Wie man sieht, hat sich der junge Mann von der Regierung, der sich erst im letzten Absatz eindeutig als derjenige herausstellt, der Damázio beleidigt haben könnte, mit all den anderen in der Folge gesagten Dingen vermischt und verschwindet vom Horizont der Vermutungen des Arztes, der sich selbst als potenzielles Opfer des Jagunço sieht.

Die von Damázio provozierte Unterbrechung endet mit der direkten Interpellation, dass der Arzt antworten soll. Wir sind jetzt bereits am Ende der Geschichte. Nur in Zeile 108, von insgesamt 150 in der hier verwendeten Ausgabe ${ }^{50}$, kann der Arzt endlich beginnen zu antworten, noch ohne den Verdacht zu verwerfen, dass ihn jemand intrigiert hat, dass von dieser Antwort sein Leben abhängen könnte. Sein nervlicher Zustand entspricht dem, dass er jetzt derjenige ist, der die Antwort aufschiebt, wie jemand, der auf Eierschalen läuft und darüber nachdenkt, was er sagen könnte. Die Angst zeigt ihre Wirkung. Er wiederholt das berüchtigte Wort, späht «wie hilfesuchend ${ }^{51}$ nach den drei schweigenden Reitern, um erst dann die Fassung wiederzugewinnen, in der Lage, sich den Unannehmlichkeiten zu entziehen: «Ich brauchte mich nur zu enthemmen». ${ }^{52}$ Erst hier beginnt der gelehrte Pedro Malasartes zu handeln, indem er mit der doppelten Bedeutung des Wortes operiert und die beleidigende (und offensichtliche) Bedeutungsnuance aus-

47 Ebda, S. 12. Übersetzung Meyer-Clason, S. 15.

48 Ebda, S. 11. Übersetzung Meyer-Clason, S. 14.

49 Ebda.

50 Gemeint ist die portugiesische Originalausgabe. (Anm. d. Üb.)

51 Rosa: Primeiras Estórias, S. 12. Übersetzung Meyer-Clason, S. 15.

52 Ebda. 
klammert, während er gleichzeitig die lobende (und wenig geläufige) Note hervorhebt, mit dem Ziel, den Jagunço zu besänftigen und die ihm unmittelbar bevorzustehen scheinende Gewalt abzuweisen. Dem Ziel dessen, der seine eigene Haut zu retten weiß.

Diese allgemeine Bewegung der Handlung zeigt, dass Damázio sein Spiel dem Arzt auferlegt. Vielleicht ist er der erste Pedro Malasartes, derjenige, der das Treffen zum beabsichtigten Ende führt. Kurz gesagt: Vielleicht möchte Damázio betrogen werden und vielleicht provoziert er diese Täuschung. Die Frage, die wir hier stellen müssen, lautet: Und warum sollte er so etwas wollen?

Und die Antwort ist gar nicht mal so schwierig. Damázio erlebt in diesem Moment eine neue und ziemlich heikle Situation. ${ }^{53}$ Es ist die Ankunft eines <jungen Mannes von der Regierung in dieser Wildnis, die darauf hindeutet, dass eine moderne Logik, die die unpersönliche Anwendung der Justiz impliziert, dort eingeführt wird. Auf der anderen Seite ist seine eigene Identifikation an die Logik des Mandonismo ${ }^{54}$ gebunden, dessen Tage gezählt sein können, der aber immer noch Macht innehat. Immerhin stellt er sich als «Damázio von den Siqueiras` vor, nicht als Damázio Siqueira, also als ein Individuum, welches durch die Verbindung mit einer Familie nicht über Blutsbande, sondern über Befehlsgewalt definiert wird: Er ist der Jagunço der Siqueiras.

Oder präziser gesagt, es existieren zwei Systeme nebeneinander, die Menschen wie Damázio zwingen, Gott und dem Teufel gemeinsam Rechenschaft abzulegen. Der Jagunço lebt auf Messers Schneide. Die so genannte archaische Ethik erhält sich noch immer aufrecht und sie hindert ihn daran, eine Beleidigung einfach auf sich beruhen zu lassen, verwickelt in die gleiche Ethik, die die berühmte finale Auseinandersetzung zwischen Matraga und Joãozinho Bem-Bem in «A hora e a vez de Augusto Matraga» (dt. Titel «Die Stunde und Umkehr des Augusto Matraga», Anm. d. Üb. $)^{55}$ steuert. Dies trifft insofern zu, da er auf die Bitte, die er an ihn heranträgt, das Leben eines bestimmten Mannes zu verschonen, nicht eingehen kann, schlichtweg weil dies gegen die Regeln wäre. Aber auch die moderne Ethik, die das Auge-um-Auge ablehnt und die Vermittlung der formell konstituierten Justiz zwischen dem, der beleidigt und dem, der beleidigt wird, verlangt, ist

53 Die Texte von José Miguel Wisnik und Ana Paula Pacheco, auf die sich hier bezogen wird, entwickeln diesen Aspekt der Geschichte ausführlich.

54 Mandonismo-Begriff, der in der brasilianischen Akademie zur Definition der Ausübung von Macht durch oligarchische und personalisierte Strukturen im Laufe der Geschichte Brasiliens verwendet wird.

55 Aus João Guimarães Rosa: Sagarana: Erzählzyklus. Aus dem Portugiesischen von Curt MeyerClason. Berlin/Weimar: Aufbau-Verlag 1984. (Anm. d. Üb.) 
wirksam und unterbindet die Schikane des Jungen, der trotz allem Repräsentant des universalen Mediators ist: der Regierung.

Wenn man Damázios Motivation bedenkt, den Doktor aufzusuchen, muss man fragen: Warum sollte ein Jagunço eine Beleidigung überprüfen müssen, bevor er jemanden tötet oder verletzt? Vorerst ist der bloße Verdacht genug, oder verpflichtet ihn in der Logik der Jagunços sogar zur schnellen Rechenschaft. Selbst José Miguel Wisnik, der nicht über diese zweite Geschichte reflektiert, sagt: «Nichts in seinem gegebenen Lebenslauf würde Damázio daran hindern, ohne zu fragen zu töten». ${ }^{56}$ Im Übrigen würde es sich sogar anbieten eine vorangehende Frage zu stellen: Bezweifelt Damázio wirklich, dass das Wort beleidigend ist? Sprache hat in der Praxis viele Dimensionen, die Wörterbücher nicht erfassen können. Diese Eigenschaft wurde ihm in einem bestimmten Kontext zugeschrieben und dort in der São Ão, von denen, die mit Damázio leben, und in Bezug auf diejenigen, die daran interessiert sind, seinen Status als starkem Mann zu bewahren, in einer bestimmten Weise verstanden. Es war schwierig für den Jagunço zu beweisen, dass das Wort berüchtigt «überhaupt nicht abfällig, keineswegs kränkend $»^{57}$ ist, um die verschlüsselte Formel zu benutzen, die unser Erzähler heraufbeschwört, um in einem ersten Moment noch unentschlossen aufzuzeigen, dass daran nichts beleidigend sei. Die Verpflichtung, den jungen Mann zu töten und sich in eine komplizierte Position zu bringen, vor allem für einen Jagunço am Ende seiner Karriere, der sozusagen bereits in seiner knarzenden Hängematte liegt, wird ihm auferlegt, aber immer noch einer «von den Siqueiras` und nicht etwa wie Riobaldo ein Erbe. Damázios Motivation, nach dem Arzt zu suchen, besteht letztlich nicht darin, alle Zweifel über den verbalen Angriff auszuräumen um den Regierungsjungen reinen Gewissens zu töten (was sogar merkwürdig klingt). Sie besteht vielmehr darin, eine Rechtfertigung dafür zu finden, den Regierungsjungen nicht zu töten und trotzdem nicht den Status als Tyrann zu verlieren, der seinen Lebensunterhalt und seine soziale Stellung garantiert.

Es war notwendig, eine autoritäre, aber distanzierte Stimme aufzusuchen, die garantiert, dass es sich um einen «keineswegs kränkend[en]» Ausdruck handelt. Außerdem musste diese Stimme aus der Ferne von nahem gehört werden. Man erinnere sich daran, dass Damázio und die drei anderen Reiter bei ihrer Ankunft in Position bleiben, um sie vor dem Blick der Straße zu schützen. Wenn es schließlich einen Grund gibt, die gewalttätigen Handlungen nicht in Gang zu bringen, ist es egal, dass diese Umgebung Aufsehen erregt oder beängstigend wirkt:

56 Wisnik: $O$ famigerado, S. 181. Üb. i. Dt.: Unsere Übersetzung (Anm. d. Üb.).

57 Rosa: Primeiras Estórias, S. 12. Übersetzung Meyer-Clason, S. 15. 
Da vor meinem Haus die Straße um ein paar Meter zurückwich und der Zaun von beiden Seiten vorrückte, entstand eine Einbuchtung, eine Art geschütztes Plätzchen. Dies hatte er genutzt, um die anderen an eine Stelle zu verweisen, wo sie weniger zu sehen waren, während er ihnen gleichzeitig jede Fluchtmöglichkeit nahm, abgesehen davon, daß sie durch ihre aneinandergepreßten Pferde an rascher Bewegung gehindert waren. ${ }^{58}$

Damázio befindet sich in einer dermaßen heiklen Situation, dass er sich verpflichtet, sich in eine für einen Jagunço unbequeme Position zu begeben. Schließlich würde er selbst in Schwierigkeiten geraten, wenn er flüchten wollte, da sich sein Pferd mit den anderen dreien in der Enge befindet. Und man beachte, wie der Doktor in der Stellung der Männer nur das Zeichen der Stärke des Mannes liest, was die anderen drei daran hindere, zu fliehen, ohne zu bemerken, dass er auf diese Weise auch seine eigene Flucht verhindert - was ein Zeichen dafür sein könnte, dass er während seines Besuches kein Verbrechen begehen möchte.

Was wäre denn die Funktion dieser drei Männer, «seine Gefangenen und nicht sein Gefolge» ${ }^{59}$, wenn es nicht die der Bezeugung der Antwort wäre, die ihn davor bewahren würde, diesen Mord zu begehen, den er nicht begehen will? Diese Funktion wird am Ende der Erzählung bestätigt, wenn Damázio sie entlässt, sobald der Arzt einen eindeutigen Beweis dafür gibt, dass berüchtigt nicht nur keine Beleidigung sei, sondern, dass es sich sogar um Lob handele: «Ihr könnt gehen, Gevattern. Ihr habt die gute Beschreibung gut gehört...) Dienstbeflissen zogen sie $a b . »{ }^{60}$

Die zweite Erzählung von «Berüchtigt` ist daher ein Spiegel der ersten. In der einen ist der, der mitunter wie Pedro Malasartes handelt, wie José Miguel Wisnik sagt, der Erzähler, der Arzt, der den unwissenden Jagunço überlistet. Und das ist die erste Erzählung, weil es diejenige ist, an die die Erzählstimme glaubt und das, was sie erzählen will. In der anderen ist Damázio derjenige, der den schlauen Gauner spielt, der den Arzt dazu bringt, ihm zu sagen, was er hören möchte - und dies von anderen gehört werden kann. Dies ist die zweite Erzählung, weil sie ohne das Zutun des Erzählers geschrieben wird, subtiler als es in «Nada e nossa Condição» der Fall ist, und doch in analoger Form.

An dieser Stelle gibt es noch eine weitere Vorsichtsmaßnahme zu ergreifen: nicht in die Falle zu tappen, zu denken, dass der Jagunço der Sieger ist, dass nur die zweite Erzählung existiert. Ja, es ist wahr, nachdem ihm bestätigt wurde, dass berüchtigt nicht erniedrigend ist, reagiert er «frohlockend» ${ }^{61}$, was darauf hinweist, dass er sich selbst als Sieger betrachtet - wer würde frohlocken, weil er et-

58 Ebda., S. 9. Übersetzung Meyer-Clason, S. 12.

59 Ebda.

60 Ebda., S. 12-13. Übersetzung Meyer-Clason, S. 15-16.

61 Ebda., S. 12. Übersetzung Meyer-Clason, S. 15. 
was nicht tut, was er gewohnt ist und tatsächlich gedacht hatte zu tun? Er fügt jedoch hinzu: «Weiß nicht, manchmal mein' ich, es wäre für den jungen Regierungsmann doch besser, fortzugehen, weiß nicht...» ${ }^{62} \mathrm{Ja}$, die Art und Weise, wie er dies ausspricht, zu sagen, dass es für den jungen Mann besser wäre, wegzugehen und nicht für ihn, bekräftigt seinen bedrohlichen Charakter und seinen Sieg. Aber in dieser Wiederholung des anfänglichen «weiß nicht» liegt im finalen «weiß nicht» auch etwas Melancholisches, was auf das Bewusstsein hindeutet, dass dies nicht passieren wird. Anders als bei Naziazeno, dem Protagonisten von Dyonélio Machados Os ratos ${ }^{63}$, der es vorzieht, alles zu überdenken, wenn er das Geld, das er dem Milchmann zu zahlen hat, bekommt, indem er sich verschuldet, offenbart Damázios Klage das Bewusstsein darüber, dass der Junge nicht weggeht - Wie gut, wenn es so wäre. Schließlich wird es mit der Ankunft der Regierung nun immer einen jungen Mann geben, der diese vertritt. Ist die Position des modernen Arztes angesichts der Gewalt, die Damázio jederzeit ausüben kann, instabil, so ist die Instabilität des Jagunços nicht geringer, der von jeglichem jungen Mann der Regierung bedroht wird, der, genau deshalb, weil er von der Regierung ist, nicht mit einem «Tüpfel auf dem i» aus der Welt geschafft werden kann. ${ }^{64}$ Und es ist gut, darauf zu bestehen, dass keiner besser ist als der andere, wenn man beide Erzählungen betrachtet. In beiden ist die gleiche Hintergrundhandlung, etwas Verachtenswertes, präsent: Diejenige, aus dem Anderen ein bloßes Instrument zu machen, sei es im Dienste seiner Annehmlichkeiten oder als seine Sache zum laut Lachen〉. Immer die gleiche List, die sich in der Leere bewegt und alles genau so beibehält, wie es war.

Auf diese Weise verstanden, erscheint «Famigerado» in seinen zwei Erzählungen nicht mehr als eine simple Herabsetzung des Jagunço. Ja, die Herabsetzung ist da, aber nicht als Gesamt- oder Einzeleffekt der Lektüre. Sie beschränkt sich auf den Standpunkt des Erzählers, auf die Beurteilung, die er von sich selbst und von diesem Unkultivierten macht - und auf eine gewisse Mittäterschaft, die er seinem gebildeten Bruder, dem Leser, zuschreibt, der das Urteil seiner eigenen Überlegenheit über den Jagunço weitergibt, mit dem er über den lachen kann, der nicht mehr beängstigend ist, weil er nicht mehr als ein Einfaltspinsel ist und sich so blind zeigt gegenüber der Intelligenz dieses Anderen.

Was die Erzählmethode beider Geschichten der Primeiras Estórias erlaubt, ist eine komplexe Gestaltung, die verschiedene Wertsysteme miteinander in Beziehung setzt, ohne eines der beiden auszuklammern, ohne dass die eine zur Norm

62 Ebda., S. 13. Übersetzung Meyer-Clason, S. 16.

63 Dt. wörtlich Die Ratten.

64 Rosa: Primeiras Estórias, S. 9. Übersetzung Meyer-Clason, S. 12. 
und die andere zur Abweichung erklärt wird. Stattdessen koexistieren sie und kollidieren, was den Leser davon abhält, voreilige Schlüsse zu ziehen. Beide Systeme praktizieren das Täuschungsspiel, ohne irgendein edles Motiv - wenn nicht dem Instinkt der Selbsterhaltung - um das Schurkenverhalten zu rechtfertigen. Beide Seiten von Pedro Malasarte - die beiden Seiten des Gaunertums - bleiben vollständig erhalten. Der Sympatische, der sich der Ordnung widersetzt, indem er innerhalb ihrer Bahnen agiert und so den Mangel an Integrität dieser Ordnung offenbart; und der andere, Unsympatische, der sich gerade deshalb, weil er in den Bahnen der Ordnung handelt, niemals gegen sie wendet - im Gegenteil, er lebt sehr gut mit ihr, nährt sich durch sie und bestätigt sie so nur.

Auf diese Weise gelesen enthüllen die Primeiras Estórias eine Darstellung des brasilianischen Modernisierungsprozesses in seiner Gänze, eine Koexistenz des Archaischen und des Modernen, die eine spezifisch brasilianische Synthese abhandelt, anders als anderswo gelebt. Nirgends ersetzen moderne Werte einfach archaische Werte, vorher koexistieren sie mit ihnen und stützen sie sich auf sich selbst in ständigem Widerspruch. In Brasilien hat dieser Widerspruch wohl ein spezifisches und tiefgreifendes Gesicht, dass in diesem Buch in großer Komplexität erscheint.

Die gleiche Komplexität tritt bei der Darstellung des Anderen in Erscheinung, des Universums des Analphabetismus. Man kann behaupten, dass Guimarães Rosa in den Primeiras Estórias mit verschiedenen Mitteln und einer anderen Vision eine Leistung erreicht, die mit der von Graciliano Ramos in Vidas secas ${ }^{65}$ vergleichbar ist: die Erscheinung der Welt der Analphabeten durch die gebildete Welt zu konstruieren, so dass beide intakt bleiben, in ihrer Größe sowie in ihren Begrenzungen und Kleinlichkeiten.

65 Deutscher Titel in der Übersetzung von Willy Keller: Karges Leben. (Anm. d. Üb.) 